\title{
Intriguing Role of Lysophosphatidic Acid (LPA) and its Receptor Mediated Signaling during Implantation: A Review
}

\author{
B. Madhumitha ${ }^{1}$, D. Reena $^{2}$ and S. Manokaran ${ }^{3} *$ \\ ${ }^{1}$ Department of Animal Biotechnology, Madras Veterinary College, Tamil Nadu Veterinary \\ and Animal Sciences University, Chennai - 600 007, India \\ ${ }^{2}$ Department of Clinics, Madras Veterinary College, TANUVAS, Chennai - 600 007, India \\ ${ }^{3}$ Kangayam Cattle Research Station, TANUVAS, \\ Uppupallam, Baguthampalayam, Sathyamangalam - 638 451, India \\ *Corresponding author
}

\section{A B S T R A C T}

\begin{tabular}{|l|}
\hline Ke y w o r d s \\
Lysophosphatidic \\
acid (LPA), \\
Assisted \\
Reproductive \\
Technology (ART), \\
Recurrent \\
implantation failure \\
(RIF), Autotaxin \\
\hline Article Info \\
\hline $\begin{array}{l}\text { Accepted: } \\
\text { 18 May } 2020 \\
\text { Available Online: } \\
\text { 10 June } 2020\end{array}$ \\
\hline
\end{tabular}

Regardless of major advances in medical technologies many Assisted Reproductive Technology (ART) experience recurrent implantation failures (RIF). Lysophosphatidic acid (LPA) signaling cross talks between the mother and the implanted embryo at a very early stage of gestation to enhance endometrium receptivity. LPA is a simple water soluble phospholipid that arbitrates varied biological functions like proliferation, migration and activation of various intracellular signaling pathways in diverse cell types. LPA also has a chief role to play in human and animal reproductive processes including luteolysis, endometrium and ovarian function, estrous cycle regulation, embryo development and implantation, placentation and decasualization with the help of receptor (LPAR1-6) mediated LPA signaling. Further, autotaxin a LPA producing enzyme is found to be upregulated during decasualization of human endometrial stromal cells (HESC). LPA aids in the maintenance of endometrium receptivity by invigorating the expression of prostaglandin endoperoxide synthase 2 (PTGS2). LPA also increases progesterone $\left(\mathrm{P}_{4}\right)$ and prostaglandin $\mathrm{E}_{2}\left(\mathrm{PGE}_{2}\right)$ secretion and elevation in $\mathrm{PGE}_{2} / \mathrm{PGF}_{2} \alpha$ ratio. This review highlights and discusses about the current advancement in receptor mediated LPA signaling in human reproduction, ruminant reproduction pointing to bovine and sheep models, and even in porcine reproduction models and correlating it with human reproductive function.

\section{Introduction}

Despite of all the advancements in reproductive technologies, majority of the implantations are unsuccessful which becomes a very important problem in humans as nowadays women decide to delay motherhood and it ultimately causes problem later. Increasing age decreases the chances of natural fertilization and pregnancy development which ultimately increases the need for new technology like Assisted Reproductive Technology (ART) (Koot et al., 2012). Implantation process in human is astonishingly dynamic and controlled by a number of molecular and cellular events. 
Though there are many new advanced technologies are emerging, success rates of in vitro fertilization (IVF) procedures remain below 30 per cent since many years. Many molecular level studies were conducted to understand the concepts in implantation failure. It is estimated that approximately 10 per cent of women experiences recurrent implantation failure (RIF) after in vitro embryo transfers. Numerous factors including oocyte and sperm quality, genetic abnormalities of the embryo, poor endometrium receptivity, immunological disturbances in the implantation site, and some gynecologic pathologies such as endometriosis, uterine fibroids etc. leads to RIF. Embryos implantation failure forms the major rate limiting step.

Unstable maternal immune response towards the embryo causes embryo rejection in some cases ie. before establishing a contact between mother and fetal an abnormal modulation in maternal immune system occurs affecting the fetus resulting in implantation failure. Lysophosphatidic acid (LPA) is a polar phospholipid gaining momentous interest in recent years (Boruszewska et al., 2013). They exert their function with the help of six $G$ protein-coupled receptors (LPAR1-6) acting as an effective signaling molecule with varied functions on many different target tissues. LPA receptors have multiple roles to play in developmental, physiological, and pathological processes (Guo et al., 2013). This review provides a summary of LPA and receptor mediated LPA signaling and current aspects on the successful implantation, pregnancy development and also in the treatment of reproductive disorders.

\section{Implantation and problems}

Best way to verify the occurrence of implantation events after assisted reproductive technology (ART) is to evaluate the human chorionic gonadotrophin (hCG) levels in serum samples after embryo implantation (Wang et al., 2003 and Koot et al., 2011). Recurrent implantation failure (RIF) is a very challenging phenomenon to be considered for any implantation. Women's age, oocyte and sperm quality, poor uterine receptivity, parental chromosomal abnormalities, endometriosis are some of the factors that contributes to RIA (Coughlan et $a l$. , 2014).Several endometrial factors also cause implantation failures. Many molecular markers including various gene expression (Revel et al., 2011), prostaglandins (Achache et al., 2010), cell adhesion molecules (Revel et al., 2005), mucins (Dentillo et al., 2007), cytokines (Zhang et al., 2011)etc. are used to determine the ideal endometrial receptive period (Quinn et al., 2007, Quinn and Casper, 2008 and Haouzi et al., 2010). Many endometrial studies can be done by using such biomarkers and many new therapies and treatments can be elucidated for treating defective endometrial receptivity (Boomsma et al., 2009).

\section{Lysophosphatidic mediated signaling}

Lysophosphatidic acid (LPA) is a watersoluble bioactive phospholipid which acts as a signaling molecule. LPA is found to be present in serum(Sano et al., 2002), plasma (Woclawek-Potocka et al., 2009), follicular fluids, ascites (Tokumura et al., 2007), neurons(Fukushima et al., 2000), ovarian cells (Eder et al., 2000)and other cell types. LPA acts as autocoid and exerts its function by activating six high affinity G-protein coupled receptors. So far six receptors have been identified and are called as LPAR1, LPAR2, LPAR3, LPAR4, LPAR5 and LPAR6. LPA performs several developmental, pathological and physiological functions in various cell types with the help of respective receptors. Receptor mediated LPA signaling has evolved 
as a new dimension in treating several human diseases (Lin et al., 2010). LPA also has a role to play in cell adhesion, differentiation of various cell types, cell migration and invasion, cell proliferation, cytoskeletal rearrangement (Moolenaar, 1995), cellular interactions (Fukushima et al., 2002), cell morphogenesis (Van Meeteren and Moolenaar, 2007) and sometimes even in cell tumorigenesis (Kim et al., 2006). Many studies have been conducted to find the correlation between LPA and female reproductive physiology (Budnik et al., 2002) and it was found that LPA signaling pathway has an influence on embryo implantation (Zhang et al., 2005).

\section{LPA signaling in human reproductive tract}

The impact of LPA on the female reproductive system has been studied for about 30 years (Liszewska et al., 2009 and Woclawek-Potocka et al., 2010). Among the six receptors present, in human it was found that there was an increase in receptor 3 expression during the early and late secretory phase and their expression decreases during mid-secretory phase. LPA and their receptors had been documented to be present in uterus, ovary and placenta (Chen et al., 2010 and Guo et al., 2013). LPA mainly influences the female reproductive function (Fig. 1) and this could be clearly understood by the presence of elevated level of LPA producing autotaxins in females (Im Kim et al., 2004 and Chen et $a l ., 2008)$.Ovarian steroids mainly estrogen and progesterone controls the human endometrial stromal cells (HESCs) during the luteal phase of menstrual cycle in human.In human LPA level increases between weeks 5 and 40 of pregnancy (Tokumura et al., 2002). During the decidualization process these cells undergoes proliferation, migration and differentiation, thus proper decidualization is important for proper implantation and successful pregnancy. During decidualization,
LPA receptors 1 and 6 were highly expressed and autotaxin enzyme level was also found to be elevated in HESCs. A recent study suggests that LPA receptor signaling is very important during human endometrium decidualization and implantation (Hanoune et al., 2005). In human follicles, oocytes and granulose cells contains lysophospholipase DAutotaxin which helps in the production of lysophosphatidic acid from lysophosphatidylcholine. When compared to women with natural cycle women receiving ovarian stimulation tends to have increased autotaxin activity. LPA signaling plays many important roles during ovulation by causing up regulation of IL-8 and IL-6 which ultimately stimulates the formation of new vessels in corpus luteum which is very important for women undergoing invitro fertilization (Jeong et al., 2016). LPA signaling is also employed in the maintenance of human pregnancy.

During pregnancy, level of serum LPA was found to be increased from both placenta and fetus. Certain studies also claim that LPA may be involved in the initiation and regulation of labour. During the beginning of labour, endometrium contraction efficiency may be increased by LPA by the formation of stress fibres in human myometrial cells. When implantation occurs in women with endometriosis LPAR3 expression tend to be decreased during the mid and late secretory phase. Thus, by relating the decreased LPAR3 expression along with other endometrium receptivity biomarkers, $\mathrm{P}_{4}$ resistance connected to endometriosis can be well explained. In addition to all the positive impacts LPA mediated signaling can also cause adverse effects during human pregnancy. Abnormal levels of LPA causes high arterial blood pressure and also vasoconstriction ultimately affecting the pregnancy. LPA in human reproductive tissues maintains ovarian cycle, helps in 
pregnancy development as well as takes care of various abnormalities in the female reproductive tract. LPA is also engaged in the direct maintenance of fetus by controlling embryo implantation and vascularization. However, for understanding the role of LPA in human reproduction, many animal models have been developed and studied in order to overcome the ethical restrictions associated with the research on human tissues(Lebovitz and Orvieto, 2014).

\section{LPA signaling in sheep reproductive system}

In ovine, endometrium conditions during early pregnancy were well studied and LPA was detected in the endometrium and conceptus of uteri during the early stage of pregnancy in ewes. Any kind of embryo implantation defects can be repaired by prostaglandin administration and cPLA-2, PTGS2, and LPAR3-mediated signaling has a major role to play. In ovine, during d 10 blastocyst which is sphericalis transformed into a tubular form on d 11-12, and on d 1316 it forms a filamentous conceptus this expansion leading to a $15 \mathrm{~cm}$ long ovine conceptus. This is mainly due to the rapid expansion of the trophectoderm initiating the implantation phases successively leading to transient connection and solid adhesion by $\mathrm{d}$ 16-17. In the sheep conceptus, trophectoderm cells were known as a source of the autotaxin.

Autotaxin and LPA levels are found to have parallel regulation and were low on d 12-14, their levels elevated on $d$ 15-16.LPA receptors LPAR1 and LPAR3 transcripts levels were at peak on $\mathrm{d} 14$ and the highest level of protein for both receptors was observed at d 17. Unlike LPAR1 which was confined in cellular membranes and nuclear compartments of the trophectoderm cells, LPAR3 was present only in membranes (Liszewska et al., 2009). It was observed that $\operatorname{ERK}(1 / 2)$ MAPK was activated after LPAR1-
3 activation. In ovine conceptus, LPAmediated signaling plays a vital role mainly at the time of embryo implantation. LPA increases the release of prostaglandin $\mathrm{F}_{2}$ and $\mathrm{E}_{2}$, without any major modifications to cytosolic phospholipase $\mathrm{A}_{2}$ and prostaglandin synthase-2 expression. Using an ovine trophectoderm cell line it was proved that LPA improved the PTGS2 expression and prostaglandin synthesis. Hence it can be concluded that the release of prostaglandin which is mainly induced by LPA mediated signaling is perhaps due to arachidonic acid mobilization. By the combined action of strong expression of PTGS2 enzyme and LPARs there is a massive increase in the synthesis of prostaglandins due to the elevated level of LPA in the uterine lumen.

\section{LPA signaling in bovine reproductive system}

Since bovine reproduction characteristics are found to be somewhat similar to that of human ovarian physiology, pregnancy maintenance, embryo implantation and development and any other assisted reproductive techniques (Bettegowda et al., 2008), few studies were conducted to understand the role of LPA in bovine uterus. In addition bovine model has a greater availability of biological material compared to studies in human. In bovine LPA was produced locally and released from its endometrium. LPA was also detected in minute concentrations in the bovine corpus lutem (CL) throughout the estrous cycle and during early pregnancy (Kowalczyk-Zieba et al., 2012). In bovine uteri prostaglandins are very important for estrous cycle regulation and maintenance of early pregnancy. Maintenance of CL, $\mathrm{P}_{4}$ secretion, maintenance of uterine receptivity is very important for successful implantation which could be made possible by attaining an optimal $\mathrm{PGF}_{2} \alpha$ to PGE2 ratio. LPA works by enhancing the activity of prostaglandin like 2 synthase, 
enzyme which converts $\mathrm{PGF}_{2} \alpha$ to $\mathrm{PGE}_{2}$.

Total LPA level in the uterine vein blood plasma was found to be significantly higher when compared to that of the jugular vein plasma on days 17-19 of the estrous cycle for $24 \mathrm{~h}$. PLD2 and PLA2G1B gene expression during estrus cycle and early was studied showing that PLD2 expression was stable during estrous cycle and early pregnancy but PLA2G1B expression was higher on days 1719 of pregnancy claiming that LPA maybe synthesized during all stages of the estrous cycle and pregnancy and preferably higher on d17-19 of pregnancy (Woclawek-Potocka., 2009).

Table.1 LPA receptors employed in various reproduction functions of different animals

\begin{tabular}{|c|c|c|c|c|}
\hline Sl. No & Animals & Receptors & Roles & Reference \\
\hline 1 & Human & $\begin{array}{l}\text { LPAR1 \& } \\
\text { LPAR3 }\end{array}$ & $\begin{array}{l}\text { Aids in HESC decidualization and } \\
\text { maintenance of progesterone level }\end{array}$ & $\begin{array}{l}\text { Brunnert et al., } \\
\text { (2014) }\end{array}$ \\
\hline 2 & Bovine & LPAR1 & $\begin{array}{l}\text { Modulates prostaglandin }(\mathrm{PG}) \\
\text { synthesis in the murine } \\
\text { endometrium. }\end{array}$ & $\begin{array}{l}\text { Jeong et al., } \\
\text { (2016) }\end{array}$ \\
\hline 3 & Sheep & $\begin{array}{l}\text { LPAR1 } \\
\text { LPAR3 }\end{array}$ & $\begin{array}{l}\text { Induces the release of } \\
\text { prostaglandin F2a and E2. }\end{array}$ & $\begin{array}{l}\text { Liszewska et al., } \\
\text { (2009) }\end{array}$ \\
\hline 4 & Porcine & LPAR3 & $\begin{array}{l}\text { Increases uterine endometrial } \\
\text { expression of PTGS2. }\end{array}$ & $\begin{array}{l}\text { Woclawek- } \\
\text { Potocka et al., } \\
(2009)\end{array}$ \\
\hline
\end{tabular}

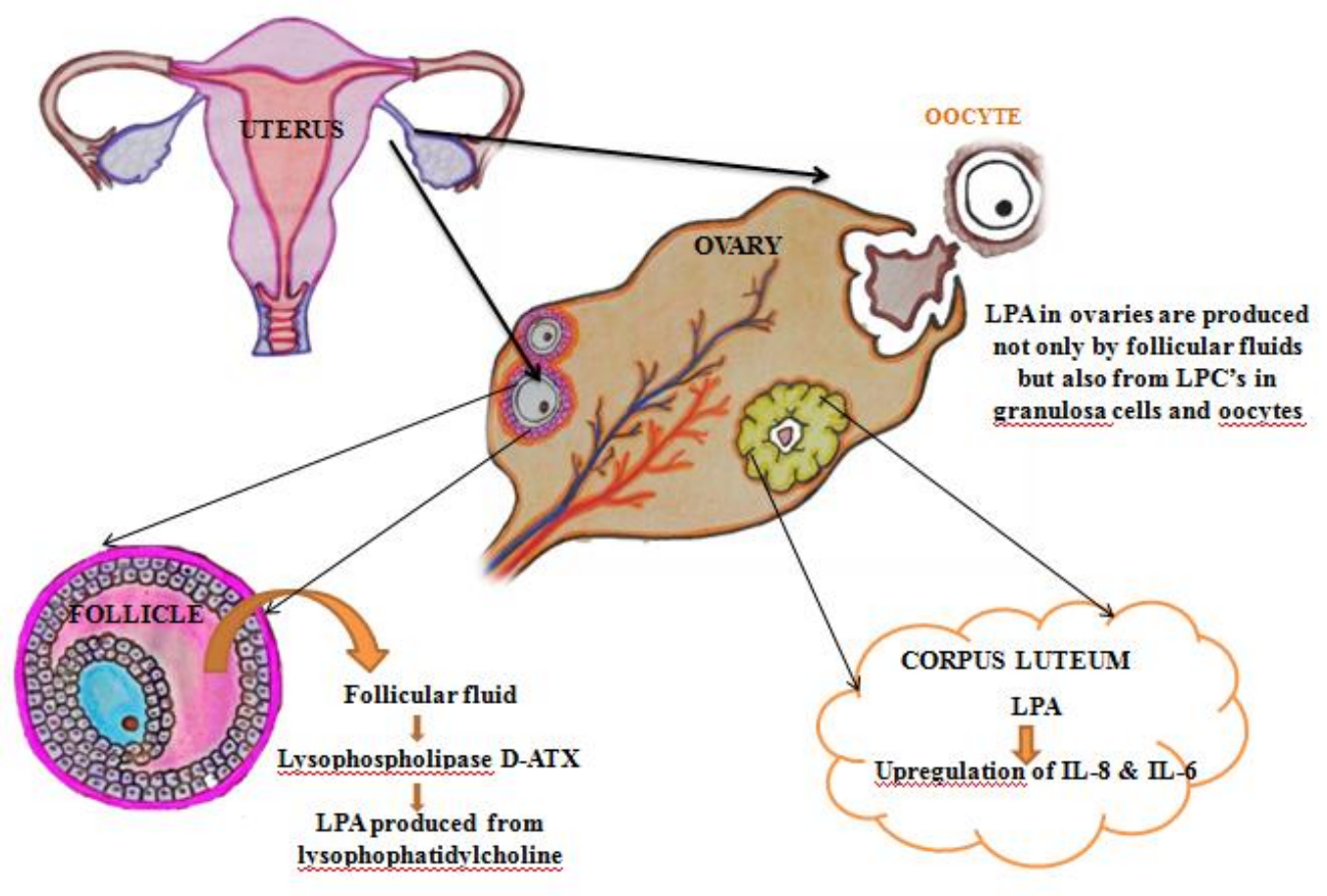

Figure.1 LPA signaling in human reproductive tract 
Autotaxin a major LPA producing enzyme and PLA2 expression were studied in bovine ovary which showed the chance of LPA synthesis in bovine follicles. In bovine endometrium during estrous cycle and early pregnancy correlation between prostaglandin synthesizing enzymes (PGES and PGFS) mRNA and LPA receptor LPAR1 expression were studied showing that PGFS mRNA expression was found to be higher on days 8 10 than on days 17-19 of pregnancy. LPA also induces autocrine and paracrine actions on the bovine endometrium, CL, and the follicle. In the bovine endometrium LPA receptor mediated signaling induce $\mathrm{PGE}_{2}$ and inhibits $\mathrm{PGF}_{2}$ aactions and in CL, LPA stimulates $\mathrm{P}_{4}$ secretion through stimulation of $3 \beta$ HSD (Sahmi et al., 2004) augments IFN $\tau$ dependent stimulation of ISG15 and OAS1 expression. LPA suppresses TNF $\alpha$ and IFN $\gamma$ (Abbas et al., 2000 and Sakumoto et al., 2000). LPA stimulates estrogen production and FSH action in bovine ovarian follicle by increasing the genes responsible for the expression of the FSHR and $17 \beta$-HSD, which sequentially relates for the involvement of LPA in bovine ovarian follicle development and differentiation.

\section{LPA signaling in porcine reproductive system}

Many studies were conducted in pigs showing that LPA and its receptor system are present at the maternal conceptus interface. Just like in all other mammals pigs also require proper peri implantation period is very important for successful pregnancy during which a wellsynchronized communication between the conceptus and the maternal uterus must be established (Aplin and Kimber, 2004 and Bazer et al., 2012). LPA elevates prostaglandin endoperoxide synthase 2 (PTGS2) in the uterine through LPAR3. Effect of LPA in porcine conceptuses was studied with the help of porcine trophectoderm (pTr) cell line isolated from Day 12 conceptuses and it was found that activation of ERK1/2-P90RSK-RPS6 and P38 pathways are involved(Bettegowda et al., 2008).

For a successful embryo implantation in human endometrium complex collaboration of several factors are important. In vitro studies using many animal models were conducted to reduce RIF and pregnancy failure by effectively rejecting the incompetent embryos. With the advancement of technologies genes and proteins of endometrial receptivity and pregnancy were identified. Many studies show evidences of LPA signaling significance in reproductive function. LPA with its $G$ protein-coupled receptors (LPAR 1-6) exerts diverse cellular effects influencing reproductive function of the female. LPA is proved to be synthesized during reproduction. Thus, it is very important to carefully examine the biological effects of LPA in human reproduction. Due to the ethical limitations in conducting human studies, numerous studies were conducted using various animal models. LPA modulates prostaglandin (PG) synthesis via LPA receptor 3 (LPAR3) and serve as an important factor in the maintenance of early pregnancy in cow. This review focuses on recent studies of LPA signaling that are related to human reproduction function, using cow, sheep, and pig as a relevant model to understand the influence of LPA on the human reproduction function.

\section{References}

Abbas, N., L.P. Zou, S.H. Pelidou, B. Winblad and Jiezhu, Z. 2000. Protective effect of Rolipram in experimental autoimmune neuritis: protection is associated with downregulation of IFN- $\gamma$ and inflammatory chemokines as well as up-regulation of IL-4 in peripheral nervous system. Journal of Autoimmunity. 32(2): 93-99. 
Achache, H., A. Tsafrir, D. Prus, R. Reich and Revel, A. 2010. Defective endometrial prostaglandin synthesis identified in patients with repeated implantation failure undergoing in vitro fertilization. Journal of Fertility and Sterility. 94(4): 1271-1278.

Aplin, J.D. and Kimber, S.J. 2004. Trophoblastuterine interactions at implantation. Journal of Reproductive Biology and Endocrinology. 2(1): 48.

Bazer, F.W., G. Song, J. Kim, K.A. Dunlap, M.C. Satterfield, G.A. Johnson, R.C. Burghardt and $\mathrm{Wu}, \mathrm{G}$. 2012. Uterine biology in pigs and sheep. Journal of Animal Science and Biotechnology. 3(1):23.

Bettegowda, A., O.V. Patel, K.B. Lee, K.E. Park, M. Salem. J. Yao, J.J. Ireland and Smith, G.W. 2008. Identification of novel bovine cumulus cell molecular markers predictive of oocyte competence: functional and diagnostic implications. Biology of Reproduction. 179(2): 301-309.

Boomsma, C.M., A. Kavelaars, M.J. Eijkemans, K. Amarouchi, G. Teklenburg and Gutknecht, D. 2009. Cytokine profiling in endometrial secretions: a non-invasive window on endometrial receptivity. Reproductive Biomedicine Online. 18(1): 85-94.

Boruszewska, D., E. Sinderewicz, I. KowalczykZieba, D.J. Skarzynski and WoclawekPotocka, I. 2013 Influence of lysophosphatidic acid on estradiol production and follicle stimulating hormone action in bovine granulosa cells. Reproductive Biology. 13(4): 344-347.

Brunnert, D., M. Sztachelska, F. Bornkessel, N. Treder, S. Wolczynski, P. Goyal and Zygmunt, M. 2014. Lysophosphatidic acid and sphingosine 1-phosphate metabolic pathways and their receptors are differentially regulated during decidualization of human endometrial stromal cells. MHR: Basic Science of Reproductive Medicine. 20(10): 1016-1025.

Budnik, L.T. and Mukhopadhyay, A.K. 2002. Lysophosphatidic acid induced nuclear localization of protein kinase $\mathrm{C} \delta$ in bovine theca cells stimulated with luteinizing hormone. Biology of reproduction. 67(3): 935-944.
Chen, S.U., C.H. Chou, K.H. Chao, H. Lee, C.W. Lin, H.F. Lu and Yang, Y.S. 2010. Lysophosphatidic acid up-regulates expression of growth-regulated oncogene- $\alpha$, interleukin-8, and monocyte chemo attractant protein-1 in human first-trimester trophoblasts: Possible roles in angiogenesis and immune regulation. Endocrinology. 151(1): 369-379.

Chen, S.U., C.H. Chou, H. Lee, C.N. Ho, C.W. Lin and Yang, W.S. 2008. Lysophosphatidic acid up-regulates expression of interleukin- 8 and -6 in granulosa-lutein cells through its receptors and nuclear factor- $\kappa \mathrm{B}$ dependent pathways: implications for angiogenesis of corpus luteum and ovarian hyperstimulation syndrome. The Journal of Clinical Endocrinology andMetabolism. 93: 935943.

Coughlan, C., W. Ledger, Q. Wang, F. Liu, A. Demirol, T. Gurgan and Li, T.C. 2014. Recurrent implantation failure: Definition and management. Reproductive BioMedicine Online. 28(1): 14-38.

Dentillo, D.B., F.R. Souza, J. Meola, G.S. Vieira, M.E. Yazlle, L.R. Goulart and Martelli, L. 2007. No evidence of association of MUC1 genetic polymorphism with embryo implantation failure. Brazilian Journal of Medical and Biological Research. 40(6): 793-797.

Eder, A.M., T. Sasagawa, M. Mao, J. Aoki and Mills, G.B. 2000. Constitutive and lysophosphatidic acid (LPA)-induced LPA production: role of phospholipase $\mathrm{D}$ and phospholipase $\mathrm{A}_{2}$. Clinical Cancer Research. 6(6): 2482-2491.

Fukushima, N., J.A. Weiner and Chun, J. 2000. Lysophosphatidic acid (LPA) is a novel extracellular regulator of cortical neuroblast morphology. Developmental Biology. 228(1): 6-18.

Fukushima, N., J.A. Weiner and Contos, J.J.A. 2002. Lysophosphatidic acid influences themorphology and motility of young, postmitotic cortical neurons. Molecular and Cellular Neuroscience. 20: 271-282.

Guo, H., F. Gong, K.L. Luo and Lu, G.X. 2013. Cyclic regulation of LPA3 in human endometrium. Archives of Gynecology and 
Obstetrics. 287(1): 131-138.

Guo, J.Y., B. Xia and White, E. (2013). Autophagy-Mediated Tumor Promotion. Cell, 155(6): 1216-1219.

Hanoune, J., X. Ye, K. Hama and Contos, J.J. 2005. LPA3-mediated lysophosphatidic acid signalling in embryo implantation and spacing. Medecine Therapeutique/ Medecine de la Reproduction, 7(4): 235.

Haouzi, D., S. Assou, C. Dechanet, T. Anahory, H. Dechaud and De Vos, J. 2010. Controlled ovarian hyperstimulation for in vitro fertilization alters endometrial receptivity in humans: protocol effects. Biology of Reproduction. 82(4): 679-686.

Im Kim J., E.J. Jo, H.Y. Lee, H.K. Kang, Y.N. Lee and Kwak, J.Y. 2004. Stimulation of early gene induction and cell proliferation by lysophosphatidic acid in human amnionderived WISH cells: role of phospholipase D-mediated pathway. Biochemical Pharmacology. 68(2): 333-340.

Jeong, W., H. Seo, Y. Sung, H. Ka, G. Song and Kim, J. 2016. Lysophosphatidic acid (LPA) receptor 3-mediated LPA signal transduction pathways: A possible relationship with early development of periimplantation porcine conceptus. Biology of Reproduction, 94(5): 104.

Kim, K.S., S. Sengupta, M. Berk, Y.G. Kwak, P.F. Escobar, J. Belinson, S.C. Mok and $\mathrm{Xu}, \quad \mathrm{Y}$. 2006. Hypoxia enhances lysophosphatidic acid responsiveness in ovarian cancer cells and lysophosphatidic acid induces ovarian tumor metastasis in vivo. Cancer Research. 66(16): 7983-7890.

Koot, Y.E., C.M. Boomsma, M.J. Eijkemans, E.G. Lentjes and Macklon,N.S. 2011. Recurrent pre-clinical pregnancy loss is unlikely to be a 'cause' of unexplained infertility. Human Reproduction. 26(10): 2636-2641.

Koot, Y., G. Teklenburg, M. Salker, J. Brosens and Macklon, N. 2012. Molecular aspects of implantation failure. Biochim. Biophys. 1822: 1943-1950.

Kowalczyk-Zieba, I., D. Boruszewska, J.S. Saulnier-Blache, L.L. Da Costa, K. Jankowska, D.J. Skarzynski and Woclawek-Potocka, I. 2012. Lysophosphatidic acid action in the bovine corpus luteum: An in vitro study. Journal of
Reproduction and Development, 58(6): 661-671.

Lebovitz, O. and Orvieto, R. 2014. Treating patients with "thin" endometrium-an ongoing challenge. Gynecological Endocrinology. 30(6): 409-414.

Lin, M.E., D.R. Herr and Chun, J. 2010. Lysophosphatidic acid (LPA) receptors: signaling properties and disease relevance. Prostaglandins and Other Lipid Mediators. 91: 130-138.

Liszewska, E., P. Reinaud, E. Billon-Denis, O. Dubois, P. Robin and Gilles, C. 2009. Lysophosphatidic acid signaling during embryo development in sheep: involvement in prostaglandin synthesis. Endocrinology. 150(1): 422-434.

Moolenaar W.H., 1995. Lysophosphatidic acid, a multifunctional phospholipid messenger. The Journal of Biological Chemistry. 270: 12949-12952.

Quinn, C.E. and Casper, R.F. 2008. Pinopodes: a questionable role in endometrial receptivity. Human Reproduction Update. 15(2): 229236.

Quinn, C., E. Ryan, E.A. Claessens, E. Greenblatt, P. Hawrylyshyn, B. Cruickshank, T. Hannam, C. Dunk and Casper, R.F. 2007. The presence of pinopodes in the human endometrium does not delineate the implantation window. Fertility and Sterility. 87(5): 1015-1021.

Revel, A., H. Achache, J. Stevens, Y. Smith and Reich, R. 2011. MicroRNAs are associated with human embryo implantation defects. Human Reproduction. 26: 2830-2840.

Revel A., A. Helman, M. Koler, A. Shushan, O. Goldshmidt, E. Zcharia, H. Aingorn and Vlodavsky, I. 2005. Heparanase improves mouse embryo implantation. Fertility and Sterility. 83(3): 580-586.

Sahmi, M., E.S. Nicola, J.M. Silva and Price, C.A. 2004. Expression of $17 \beta$-and $3 \beta$ hydroxysteroid dehydrogenases and steroidogenic acute regulatory protein in non-luteinizing bovine granulosa cells in vitro. Molecular and Cellular Endocrinology. 223(1-2): 43-54.

Sakumoto, R., B. Berisha, N. Kawate, D. Schams and Okuda, K. 2000. Tumor necrosis factor- $\alpha$ and its receptor in bovine corpus 
luteum throughout the estrous cycle. Biology of Reproduction, 62(1): 192-199.

Sano, T., D.L. Baker, T. Virag, A. Wada, Y. Yatomi, T. Kobayashi, Y. Igarashi and Tigyi, G.J. 2002. Multiple mechanisms linked to platelet activation result in lysophosphatidic acid and sphingosine-1phosphate generation in blood. Journal of Biological Chemistry. 277(24): 2119721206.

Tokumura, A., T. Kume, K. Fukuzawa, M. Tahara, K. Tasaka, J. Aoki, H. Arai, K. Yasuda and Kanzaki, H. 2007. Peritoneal fluids from patients with certain gynecologic tumor contain elevated levels of bioactive lysophospholipase D activity. Life Sciences, 80(18): 1641-1649.

Tokumura, A., Y. Kanaya, M. Miyake, S. Yamano, M. Irahara and Fukuzawa, K. 2002. Increased production of bioactive lysophosphatidic acid by serum lysophospholipase D in human pregnancy. Biology of Reproduction. 67(5): 13861392.

Van Meeteren, L.A. and Moolenaar, W.H. 2007. Regulation and biological activities of the autotaxin-LPA axis. Progress in Lipid Research. 46(2): 145-160.

Woclawek-Potocka, I., I. Kowalczyk-Zieba and Skarzynski, D.J. 2010. Lysophosphatidic acid action during early pregnancy in the cow: in vivo and in vitro studies. Journal of Reproduction and Development. 56(4):
411-420.

Woclawek-Potocka, I., J. Komiyama, J.S. Saulnier-Blache, E. Brzezicka, M.M. Bah, K. Okuda and Skarzynski, D.J. 2009. Lysophosphatic acid modulates prostaglandin secretion in the bovine uterus. Reproduction. 137(1): 95-105.

Wang, X., C. Chen, L. Wang, D. Chen, W. Guang and French, J. 2003. Reproductive Health Study Group. Conception, early pregnancy loss, and time to clinical pregnancy: a population-based prospective study. Journal of Fertility and Sterility. 79(3): 577-584.

Woclawek-Potocka, I., E. Brzezicka and Skarzynski, D.J. 2009. Lysophosphatic acid modulates prostaglandin secretion in the bovine endometrial cells differently on days 8-10 of the estrous cycle and early pregnancy. Journal of Reproduction and Development. 55(4): 393-939.

Zhang, J., Z. Chen, G.N. Smith and Croy, B.A. 2011. Natural killer cell-triggered vascular transformation: maternal care before birth. Cellular and Molecular Immunology. 8(1): 1.

Zhang, R., M.V. Poustovoitov, X. Ye, H.A. Santos, W. Chen, S.M. Daganzo and Adams, P.D. 2005. Formation of MacroH2A-Containing SenescenceAssociated Heterochromatin Foci and Senescence Driven by ASF1a and HIRA. Developmental Cell. 8(1): 19-30.

\section{How to cite this article:}

Madhumitha. B., D. Reena and Manokaran. S. 2020. Intriguing Role of Lysophosphatidic Acid (LPA) and its Receptor Mediated Signaling during Implantation: A Review. Int.J.Curr.Microbiol.App.Sci. 9(06): 2381-2389. doi: https://doi.org/10.20546/ijcmas.2020.906.292 